\title{
A Coupled Discrete Unified Gas-Kinetic Scheme for Convection Heat Transfer in Porous Media
}

\author{
Peiyao Liu ${ }^{1}$, Peng Wang ${ }^{1}$, Long $\mathrm{Jv}^{1}$ and Zhaoli Guo ${ }^{1, *}$ \\ 1 State Key Laboratory of Coal Combustion, Huazhong University of Science and \\ Technology, Wuhan, 430074, P.R. China.
}

Received 12 November 2019; Accepted (in revised version) 28 January 2020

\begin{abstract}
In this paper, the discrete unified gas-kinetic scheme (DUGKS) is extended to the convection heat transfer in porous media at representative elementary volume (REV) scale, where the changes of velocity and temperature fields are described by two kinetic equations. The effects from the porous medium are incorporated into the method by including the porosity into the equilibrium distribution function, and adding a resistance force in the kinetic equation for the velocity field. The proposed method is systematically validated by several canonical cases, including the mixed convection in porous channel, the natural convection in porous cavity, and the natural convection in a cavity partially filled with porous media. The numerical results are in good agreement with the benchmark solutions and the available experimental data. It is also shown that the coupled DUGKS yields a second-order accuracy in both temporal and spatial spaces.
\end{abstract}

AMS subject classifications: 82B40, 76S05, 76E06

Key words: Coupled discrete unified gas-kinetic scheme, generalized Navier-Stokes equations, porous media, convection heat transfer.

\section{Introduction}

Convection heat transfer in porous media has long been a subject of research due to its extensive applications in engineering, such as heat exchangers, electronic cooling instruments, and pollutant diffusion [1-4]. Over the past several decades, considerable investigations and applications have been devoted to the convection heat transfer in porous media through various traditional numerical methods, such as the finite volume method, the finite difference method, and the finite element method.

*Corresponding author. Email addresses: liupeiyao@hust.edu.cn (P. Liu), peng-wang@hust.edu.cn (P. Wang), jvlong@hust.edu.cn (L. Jv), zlguo@hust.edu.cn (Z. Guo) 
In general, the modelling of porous flow can be classified into two categories, i.e. the pore-scale model and the representative elementary volume (REV) scale model. In the pore-scale study, the detailed geometric information of the pores should be known, and each pore requires sufficient grid resolution in the simulations. Thus, the computational domain size cannot be too large in view of the limited computer resources. An alternative approach is to investigate the averaged quantities at the REV scale. At this scale, a number of models based on some semi-empirical relations have been developed. Up to date, most investigations at the REV scale are based on the Darcy law $[5,6]$ as the Reynolds number based on pore diameter is smaller than 1 [7] such that inertial effect can be ignored. For flows with larger Reynolds numbers, various extended Darcy models have been proposed. For instance, the Darcy-Brinkman model [8,9], which considers the viscous dissipation introduced by solid boundary, allows to study high-porosity porous flows, while the Darcy-Forchheimer model $[10,11]$ includes an additional nonlinear resistance term (Forchheimer term), and a generalized model was further developed in which both the Brinkman and Forchheimer effects are included $[12,13]$, such that the flows in wide range of flow regimes can be described. Based on this generalized model, a number of flow and heat transfer problems in porous media have been studied [14-16]. For example, Arpino et al. studied the transient natural convection in partially porous annuli, and clarified impacts on both porous medium properties and geometrical characteristics of the domain [17].

In the past years, several types of kinetic methods, which can be viewed as alternative numerical tools to traditional ones, have been successfully employed for porous media flows based on generalized model [18-20]. Particularly, the lattice Boltzmann method (LBM) has been recognized as a powerful tool for such flows. Guo et al. proposed a LBM with the Bhatnagar-Gross-Krook collision operator (LBGK) for flow and heat transfer at the REV scale [21], and a model with multiple-relaxation-time (MRT) model was subsequently proposed to improve the numerical stability [22,23]. In addition to the standard LBM, a finite-volume LBM was also developed for thermal flows in porous media [24]. Although the LBM models mentioned above have gained much success, some limitations still exist. For example, the computational time step and grid size are coupled, so that the flexibility of relaxation time and the numerical stability are very limited [25].

Recently, another kinetic method, a discrete unified gas-kinetic scheme (DUGKS) has been presented for both hydrodynamic and rarefied flows [26-30]. As a finite-volume method, the DUGKS can be easily implemented on non-uniform or unstructured meshes to satisfy the local accuracy requirement $[25,31,32]$. Although DUGKS was originally developed for multiscale flows beyond continuum regime, it can also be applied to continuum flows on which the LBM focuses. Under such circumstances, the DUGKS exhibits several distinctive features in comparison with LBM. In fact, several comparative studies of the standard LBM and DUGKS have been preformed systematically for laminar flows [25,33], turbulent flows [34,35], and natural convection flows [36,37] in previous work. Generally, for flows without solid boundaries, for example the decaying turbulent flow, the accuracy of standard LBM is slightly better than the DUGKS [34], while 
for flows involving solid boundaries, the DUGKS is even more accurate than the standard LBM $[25,33]$. Furthermore, owing to the semi-implicitness in the construction of gas distribution function at the cell interfaces, the stability and robustness of DUGKS are better than LBM $[25,33]$. Most importantly, benefitted from the finite-volume nature, the efficiency of DUGKS can be significantly improved by employing a non-uniform mesh according to the local accuracy requirement [25], without loss of accuracy and additional efforts. However, although DUGKS has been applied to a variety of flow problems $[28,35,36]$, no studies have been reported for flow and hear transfer in porous media. In this work, we aim to extended the DUGKS to such systems.

The rest of this paper is organized as follows. The governing equations of the generalized model for thermal flows in porous media are described in Section. 2. The kinetic model equations and the DUGKS are presented in Section 3. The model validation are given in Section 4, followed by a summary and discussion in Section 5 .

\section{Generalized model for thermal porous flows}

Under the Boussinesq assumption, the thermal flow of an incompressible fluid in a homogeneous isotropic porous medium can be described by the following generalized NavierStokes equations [21,38,39],

$$
\begin{aligned}
& \nabla \cdot \boldsymbol{u}=0, \\
& \frac{\partial \boldsymbol{u}}{\partial t}+(\boldsymbol{u} \cdot \nabla)\left(\frac{\boldsymbol{u}}{\epsilon}\right)=-\frac{1}{\rho_{f}} \nabla(\epsilon p)+v_{e} \nabla^{2} \boldsymbol{u}+\boldsymbol{F}, \\
& \sigma \frac{\partial T}{\partial t}+\boldsymbol{u} \cdot \nabla T=\nabla \cdot\left(\alpha_{m} \nabla T\right),
\end{aligned}
$$

where $u, p$, and $T$ are the volume-averaged velocity, pressure, and temperature, respectively; $v_{e}$ is the effective viscosity; $\epsilon$ is the porosity; the parameter $\sigma=\epsilon+(1-\epsilon) \rho_{s} c_{p s} / \rho_{f} c_{p f}$ is the thermal capacity ratio between the solid and fluid phases, in which $\rho_{f}\left(\rho_{s}\right)$ and $c_{p f}$ $\left(c_{p s}\right)$ are the density and specific heat of fluid (solid) phase, respectively; $\alpha_{m}=k_{m} / \rho_{f} c_{p f}$ is the effective thermal diffusivity, with $k_{m}$ being the effective thermal conductivity of porous media. The force term $\boldsymbol{F}$ denotes the total force induced by porous media and other body forces [19],

$$
\boldsymbol{F}=-\frac{\epsilon v}{K} \boldsymbol{u}-\frac{\epsilon F_{\epsilon}}{\sqrt{K}}|\boldsymbol{u}| \boldsymbol{u}+\epsilon \boldsymbol{G},
$$

where $K$ is the permeability of porous medium, $v$ is the viscosity of the fluid, and usually $v=v_{e}[40]$. The body force $G$ is given by

$$
\boldsymbol{G}=\boldsymbol{g} \beta\left(T-T_{r e f}\right)+\boldsymbol{a},
$$

where the first term denotes the buoyancy force, $g$ is the gravitational acceleration, $\beta$ is the thermal expansion coefficient, $T_{r e f}$ is the reference temperature, and the second term 
$a$ is the acceleration due to other external force. According to experimental results, the geometric function $F_{\epsilon}$ and the permeability $K$ can be expressed as [41]

$$
F_{\epsilon}=\frac{1.75}{\sqrt{150 \epsilon^{3}}}, \quad K=\frac{\epsilon^{3} d_{p}^{2}}{150(1-\epsilon)^{2}}
$$

where $d_{p}$ is the diameter of the solid spherical particle of the porous medium.

The flow governed by Eq. (2.1) is characterized by several non-dimensional parameters, including the Darcy number Da, the Prandtl number Pr, the Reynolds number Re (for mixed convection), the Rayleigh number Ra (for natural convection), and the viscosity ratio Je, which are defined as

$$
\mathrm{Da}=\frac{K}{L^{2}}, \quad \operatorname{Pr}=\frac{v_{e}}{\alpha_{m}}, \quad \operatorname{Re}=\frac{L U}{v}, \quad \operatorname{Ra}=\frac{g \beta \Delta T L^{3}}{v \alpha_{m}}, \quad \mathrm{Je}=\frac{v_{e}}{v},
$$

where $\Delta T$ is the temperature difference (characteristic temperature), $L$ and $U$ are the characteristic length and velocity, respectively.

\section{Coupled discrete unified gas-kinetic scheme}

\subsection{Kinetic model equations}

The following Boltzmann equations with Bhatnagar-Gross-Krook (BGK) collision operators are employed [36],

$$
\begin{aligned}
& \frac{\partial f}{\partial t}+\xi \cdot \nabla f=\bar{\Omega} \equiv \Omega+S \equiv \frac{f^{e q}-f}{\tau_{v}}+S \\
& \frac{\partial g}{\partial t}+\xi \cdot \nabla g=\Psi \equiv \frac{g^{e q}-g}{\tau_{c}}
\end{aligned}
$$

where $f=f(\boldsymbol{x}, \boldsymbol{\xi}, t)$ and $g=g(\boldsymbol{x}, \boldsymbol{\xi}, t)$ are gas distribution functions for velocity and temperature fields, respectively, and both $f$ and $g$ are functions of space $x$, molecular velocity $\xi$, and time $t ; f^{e q}$ and $g^{e q}$ are the equilibrium distribution functions; $\Omega$ and $\Psi$ are collision terms, with $\tau_{v}$ and $\tau_{c}$ being the relaxation times. $S$ is an external force term.

To account for the effect of porous media, the porosity $\epsilon$ and the thermal capacity ratio $\sigma$ are introduced into $f^{e q}$ and $g^{e q}$, respectively. The modified equilibrium distribution functions $f^{e q}$ and $g^{e q}$ take the following forms for low Mach number flows [21],

$$
\begin{aligned}
& f^{e q}=W_{\alpha}\left[1+\frac{\boldsymbol{\xi} \cdot \boldsymbol{u}}{R T_{0}}+\frac{(\boldsymbol{\xi} \cdot \boldsymbol{u})^{2}}{2 \epsilon\left(R T_{0}\right)^{2}}-\frac{|\boldsymbol{u}|^{2}}{2 \epsilon R T_{0}}\right], \\
& g^{e q}=W_{\beta}\left[\sigma+\frac{\boldsymbol{\xi} \cdot \boldsymbol{u}}{R T_{0}}\right]
\end{aligned}
$$


where $W_{\alpha}=\frac{\rho}{\left(2 \pi R T_{0}\right)^{D / 2}} \exp \left(\frac{|\xi|^{2}}{2 R T_{0}}\right), W_{\beta}=\frac{T}{\left(2 \pi R T_{0}\right)^{D / 2}} \exp \left(\frac{|\xi|^{2}}{2 R T_{0}}\right), D$ is the spatial dimension, $R$ is the gas constant, $T_{0}$ is the constant variance which determine the artificial sound speed of the velocity, $\rho$ and $T$ are the density and temperature of the fluid, respectively. For continuum flows, the total force term $S$ can be approximated as [42]

$$
S=-\boldsymbol{F} \cdot \nabla_{\xi} f^{e q}=\frac{\boldsymbol{F} \cdot(\boldsymbol{\xi}-\boldsymbol{u})}{R T_{0}} f^{e q} .
$$

Notice that $\int S d \xi=0$, and $\int S \xi d \xi=\rho \boldsymbol{F}$. The density, velocity and temperature $(\rho, \boldsymbol{u}$, and $T$ ) are defined as the moments of the distribution functions,

$$
\rho=\int f d \xi, \quad \rho \boldsymbol{u}=\int \xi f d \xi, \quad \sigma T=\int g d \xi .
$$

Through the Chapman-Enskog procedure (some details can be found in the Appendix), Eqs. (3.1) and (3.2) can recover the macroscopic governing equations given in Eq. (2.1) exactly under the incompressible limit, with the effective viscosity

$$
v_{e}=\tau_{v} R T_{0}
$$

and the effective thermal diffusivity

$$
\alpha_{m}=\sigma \tau_{c} R T_{0}
$$

It is noted that the Prantdl number can be written as

$$
\operatorname{Pr}=\frac{v_{e}}{\alpha_{m}}=\frac{\tau_{v}}{\sigma \tau_{c}} .
$$

Therefore, Pr can be modified by choosing appropriate $\tau_{v}$ and $\tau_{c}$.

\subsection{DUGKS for velocity field}

The DUGKS formulates a discrete form of the Boltzmann equation. As a finite-volume scheme, the computational domain is divided into a set of control volumes. First, integrating Eq. (3.1) on a control volume $V_{j}$ centered at cell $x_{j}$ from time $t_{n}$ to $t_{n+1}=t_{n}+\Delta t$ ( $\Delta t$ is the time step), the updating equation can be written as

$$
f_{j}^{n+1}-f_{j}^{n}+\frac{\Delta t}{\left|V_{j}\right|} F^{n+1 / 2}=\frac{\Delta t}{2}\left(\bar{\Omega}_{j}^{n+1}+\bar{\Omega}_{j}^{n}\right),
$$

where the midpoint rule for the integration of flux term, the trapezoidal rule for the collision term and force term are used, $F^{n+1 / 2}$ is the micro-flux across the cell interface,

$$
F^{n+1 / 2}=\int_{\partial V_{j}}(\boldsymbol{\xi} \cdot \boldsymbol{n}) f\left(\boldsymbol{x}, t_{n+1 / 2}\right) d \boldsymbol{x}
$$


where $\boldsymbol{n}$ is the unit vector normal to the cell interface. In order to convert implicit form to an explicit version in Eq. (3.10), two auxiliary distribution functions related to the original distribution function $f$ are introduced,

$$
\tilde{f}=f-\frac{\Delta t}{2} \bar{\Omega}, \quad \tilde{f}^{+}=f+\frac{\Delta t}{2} \bar{\Omega} .
$$

With these facts, evolution equation of DUGKS can be rewritten as

$$
\tilde{f}_{j}^{n+1}=\tilde{f}_{j}^{+, n}-\frac{\Delta t}{\left|V_{j}\right|} F^{n+1 / 2} .
$$

Above all, we can explicitly track the evolution of $\tilde{f}$ instead of the original one. Since the collision term $\Omega$ is mass and momentum conservative, the fluid density $\rho$ and the velocity $\boldsymbol{u}$ can be directly computed from $\tilde{f}$ as that of $f$ given in Eq. (3.6).

The key in updating the $\tilde{f}$ is to get the micro-flux $F^{n+1 / 2}$, which can be determined by the distribution function $f\left(x_{b}, \xi, t_{n+1 / 2}\right)$ at cell interface. To this end, integrating Eq. (3.1) with a half time step $h(h=\Delta t / 2)$ along the characteristic line ended at the cell interface $\left(x_{b}\right)$, and using the trapezoidal rule for collision and force terms, we can obtain

$$
f\left(x_{b}, \xi, t_{n}+h\right)-f\left(x_{b}-\xi h, \xi, t_{n}\right)=\frac{h}{2}\left[\bar{\Omega}\left(x_{b}, \xi, t_{n}+h\right)+\bar{\Omega}\left(x_{b}-\xi h, \xi, t_{n}\right)\right] .
$$

To remove the implicity of Eq. (3.14), we introduce another two distribution functions,

$$
\bar{f}=f-\frac{h}{2} \bar{\Omega}, \quad \bar{f}^{+}=f+\frac{h}{2} \bar{\Omega} .
$$

Then Eq. (3.14) can be simplified to

$$
\bar{f}\left(x_{b}, \xi, t_{n}+h\right)=\bar{f}^{+}\left(x_{b}-\xi h, \xi, t_{n}\right) .
$$

For smooth flows, $\bar{f}^{+}\left(\boldsymbol{x}_{\boldsymbol{b}}-\boldsymbol{\xi} h, \xi, t_{n}\right)$ can be approximated by its Taylor expansion around $x_{b}$,

$$
\bar{f}^{+}\left(x_{b}-\xi h, \xi, t_{n}+h\right)=\bar{f}^{+}\left(x_{b}, \xi, t_{n}\right)-h \xi \cdot \sigma_{b},
$$

where the gradient $\sigma_{b}=\nabla \bar{f}^{+}\left(x_{b}, \xi, t_{n}\right)$ can be approximated by linear interpolation. As a result, Eq.(3.16) can be reconstructed as

$$
\bar{f}\left(x_{b}, \xi, t_{n}+h\right)=\bar{f}^{+}\left(x_{b}, \xi, t_{n}\right)-h \xi \cdot \sigma_{b} .
$$

Again using the conservative property of $\Omega$, the conserved variables at the cell interface can be computed from $\bar{f}\left(x_{b}, \xi, t_{n}+h\right)$. From the obtained variables, the equilibrium distribution function $f^{e q}\left(x_{b}, \xi, t_{n}+h\right)$ can be determined. As such, the original distribution function $f\left(x_{b}, \xi, t_{n}+h\right)$ at cell interface can be extracted from $\bar{f}$,

$$
f\left(x_{b}, \xi, t_{n}+h\right)=\frac{2 \tau_{v}}{2 \tau_{v}+h} \bar{f}\left(x_{b}, \xi, t_{n}+h\right)+\frac{h}{2 \tau_{v}+h} f^{e q}\left(x_{b}, \xi, t_{n}+h\right)+\frac{\tau_{v} h}{2 \tau_{v}+h} S,
$$


from which the micro-flux $F^{n+1 / 2}$ can be computed from Eq. (3.11). By now, the update of the distribution function $\tilde{f}$ can be done according to Eq. (3.13). The required variables in the evolution are

$$
\begin{aligned}
\bar{f}^{+} & =\frac{2 \tau_{v}-h}{2 \tau_{v}+\Delta t} \tilde{f}+\frac{3 h}{2 \tau_{v}+\Delta t} f^{e q}+\frac{3 \tau_{v} h}{2 \tau_{v}+\Delta t} S, \\
\tilde{f}^{+} & =\frac{4}{3} \bar{f}^{+}-\frac{1}{3} \tilde{f} .
\end{aligned}
$$

As a discrete ordinate method, the remaining task in DUGKS is to discretize the velocity space. For two-dimensional flows considered in this study, the two-dimensional and nine velocity (D2Q9) model [43] is employed in velocity field. As a result, the molecular velocity is

$$
\xi_{i}= \begin{cases}(0,0), & i=0, \\ (\cos [(i-1) \pi / 2], \sin (i-1) \pi / 2) c, & i=1-4, \\ (\cos [(i-5) \pi / 2+\pi / 4], \sin (i-5) \pi / 2+\pi / 4) \sqrt{2} c, & i=5-8,\end{cases}
$$

where $c=\sqrt{3 R T_{0}}$ is the model speed of sound. The corresponding weight coefficients $\left\{\omega_{i} \mid i=0,1, \cdots, 8\right\}$ are $\omega_{0}=\frac{4}{9}, \omega_{1-4}=\frac{1}{9}, \omega_{5-8}=\frac{1}{36}$. With this velocity space, the discrete equilibrium distribution function can be expressed as

$$
f_{i}^{e q}=\omega_{i} \rho\left[1+\frac{\xi_{i} \cdot \boldsymbol{u}}{R T_{0}}+\frac{\left(\boldsymbol{\xi}_{i} \cdot \boldsymbol{u}\right)^{2}}{2 \epsilon\left(R T_{0}\right)^{2}}-\frac{|\boldsymbol{u}|^{2}}{2 \epsilon R T_{0}}\right] .
$$

The macroscopic quantities $\rho$ and $\boldsymbol{u}$ are calculated by

$$
\rho=\sum_{i=0}^{8} \tilde{f}_{i}, \quad \rho \boldsymbol{u}=\sum_{i=0}^{8} \tilde{\xi}_{i} \tilde{f}_{i}+\frac{\Delta t}{2} \rho \boldsymbol{F} .
$$

Since the linear and non-linear force given in Eq. (2.2) contain the velocity term, Eq. (3.24) represents a nonlinear equation for the velocity. Due to this situation, the fluid velocity $u$ can be explicitly calculated by a temporal velocity $v$, i.e.

$$
\boldsymbol{u}=\frac{\boldsymbol{v}}{c_{0}+\sqrt{c_{0}^{2}+c_{1}|\boldsymbol{v}|}}
$$

where

$$
\rho v=\sum_{i=0}^{8} \xi_{i} \tilde{f}_{i}+\frac{\Delta t}{2} \epsilon \rho G, \quad c_{0}=\frac{1}{2}\left(1+\epsilon \frac{\Delta t}{2} \frac{v}{K}\right), \quad c_{1}=\epsilon \frac{\Delta t}{2} \frac{F_{\epsilon}}{\sqrt{K}} .
$$




\subsection{DUGKS for temperature field}

For the update of the temperature fields, similar treatments as the DUGKS for the velocity field can be implemented on Eq. (3.2). Firstly, Eq. (3.2) is integrated at the same control volume $V_{j}$ in a time step, and the same integration rules are adopted, one can get the updating equation

$$
g_{j}^{n+1}-g_{j}^{n}+\frac{\Delta t}{\left|V_{j}\right|} \bar{F}^{n+1 / 2}=\frac{\Delta t}{2}\left[\Psi_{j}^{n+1}+\Psi_{j}^{n}\right],
$$

where the micro-flux $\bar{F}^{n+1 / 2}$ across the cell interface is

$$
\bar{F}^{n+1 / 2}=\int_{\partial V_{j}}(\boldsymbol{\xi} \cdot \boldsymbol{n}) g\left(\boldsymbol{x}, t_{n+1 / 2}\right) d \boldsymbol{x} .
$$

Two auxiliary distribution functions are introduced to make Eq. (3.27) explicit,

$$
\tilde{g}=g-\frac{\Delta t}{2} \Psi, \quad \tilde{g}^{+}=g+\frac{\Delta t}{2} \Psi .
$$

Then the evolution equation of Eq. (3.27) can rewritten as

$$
\tilde{g}_{j}^{n+1}=\tilde{g}_{j}^{+, n}-\frac{\Delta t}{\left|V_{j}\right|} \bar{F}^{n+1 / 2}
$$

Based on the conservative property of $\Psi$, the conserved variable can be directly computed from $\tilde{g}$ as that of $g$ given in Eq. (3.6).

The next task is to evaluate the microflux $\bar{F}^{n+1 / 2}$. We again integrate Eq. (3.2) within a half time step $h$ along the characteristic line with the end point $x_{b}$ at the cell interface, and adopt the trapezoidal rule to evaluate the collision term, one can obtain

$$
g\left(x_{b}, \xi, t_{n}+h\right)-g\left(x_{b}-\xi h, \xi, t_{n}\right)=\frac{\Delta t}{2}\left[\Psi\left(x_{b}, \xi, t_{n}+h\right)+\Psi\left(x_{b}-\xi h, \xi, t_{n}\right)\right] .
$$

Also another two new distribution functions are introduced to remove the implicity in the above equation,

$$
\bar{g}=g-\frac{h}{2} \Psi, \quad \bar{g}^{+}=g+\frac{h}{2} \Psi .
$$

Then Eq. (3.31) can be rewritten as

$$
\bar{g}\left(x_{b}, \xi, t_{n}+h\right)=\bar{g}^{+}\left(x_{b}-\xi h, \xi, t_{n}\right) .
$$

After the Taylor expansion of $\bar{g}^{+}\left(x_{b}-\xi h, \xi, t_{n}\right)$ made around the cell interface $x_{b}$, Eq. (3.33) can be rewritten as

$$
\bar{g}\left(x_{\boldsymbol{b}}, \boldsymbol{\xi}, t_{n}+h\right)=\bar{g}^{+}\left(\boldsymbol{x}_{\boldsymbol{b}}, \boldsymbol{\xi}, t_{n}\right)-h \boldsymbol{\xi} \cdot \nabla \bar{g}^{+}\left(\boldsymbol{x}_{\boldsymbol{b}}, \boldsymbol{\xi}, t_{n}\right) .
$$

Based on Eq. (3.32), the temperature $T$ related to $\bar{g}$ at cell interface can be obtained like that of $g$ given in Eq. (3.6). Together with the conserved variables in velocity field, the 
equilibrium distribution function $g^{e q}\left(x_{b}, \xi, t^{n}+h\right)$ can be fully determined. Therefore, the original distribution function $g\left(x_{b}, \xi, t_{n}+h\right)$ can be obtained,

$$
g\left(x_{b}, \xi, t_{n}+h\right)=\frac{2 \tau_{c}}{2 \tau_{c}+h} \bar{g}\left(x_{b}, \xi, t_{n}+h\right)+\frac{h}{2 \tau_{c}+h} g^{e q}\left(x_{b}, \xi, t_{n}+h\right),
$$

from which the micro-flux $\bar{F}^{n+1 / 2}$ can be computed from Eq. (3.28). In the implementation of DUGKS, $\bar{g}^{+}$and $\tilde{g}$ at cell center can be effectively computed by

$$
\begin{aligned}
& \bar{g}^{+}=\frac{2 \tau_{c}-h}{2 \tau_{c}+\Delta t} \tilde{g}+\frac{3 h}{2 \tau_{c}+\Delta t} g^{e q}, \\
& \tilde{g}^{+}=\frac{4}{3} \bar{g}^{+}-\frac{1}{3} \tilde{g} .
\end{aligned}
$$

Up to this point, the discretization of the Boltzmann equation Eq. (3.2) in time and space domains is completed. Here, the following two-dimensional and five velocity model (D2Q5) is employed to discretize the velocity space,

$$
\boldsymbol{\xi}_{i}= \begin{cases}(0,0), & i=0, \\ (\cos [(i-1) \pi / 2], \sin (i-1) \pi / 2) c, & i=1-4,\end{cases}
$$

where $c=\sqrt{3 R T_{0}}$. The associated weights $\left\{\bar{\omega}_{i} \mid i=0,1, \cdots, 4\right\}$ are $\bar{\omega}_{0}=\frac{1}{3}, \bar{\omega}_{1-4}=\frac{1}{6}$, and the discrete equilibrium distribution function is

$$
g_{i}^{e q}=\bar{\omega}_{i} T\left[\sigma+\frac{\xi_{i} \cdot \boldsymbol{u}}{R T_{0}}\right] .
$$

The temperature $T$ of the fluid is

$$
\sigma T=\sum_{i=0}^{4} \tilde{g}_{i}
$$

It is noted that the D2Q9 model can also be employed to discretize the velocity space. Actually, several comparative studies of LBM with the D2Q9 and D2Q5 models have been conducted $[44,45]$, and the results showed that the D2Q5 model is adequate for the convection-diffusion equation governing the temperature. Furthermore, the equilibrium distribution of $\mathrm{D} 2 \mathrm{Q} 5$ model ignores the nonlinear velocity terms, and is thus more computational efficient. Therefore, in the present study we use the D2Q5 model for the temperature field.

\subsection{Kinetic boundary conditions}

In this subsection, we will introduce several kinds of kinetic boundaries for the velocity and temperature distribution functions. For velocity field, the bounce-back scheme is employed at the wall $\boldsymbol{x}_{w}$, which can be expressed as [36]

$$
\bar{f}\left(x_{w}, \xi_{i}, t+h\right)=\bar{f}\left(x_{w},-\xi_{i}, t+h\right)+2 \rho_{w} \omega_{i} \frac{\boldsymbol{\xi}_{i} \cdot \boldsymbol{u}_{w}}{R T_{0}}, \quad \boldsymbol{\xi}_{i} \cdot \boldsymbol{n}>0,
$$


where $\boldsymbol{n}$ is the unit vector normal to the wall pointing to the cell, $\rho_{w}$ and $\boldsymbol{u}_{\boldsymbol{w}}$ are the density and velocity at the wall, respectively. For nearly incompressible flows, the $\rho_{w}$ can be approximately equal to the constant density.

The bounce-back rule for an adiabatic wall is [36]

$$
\bar{g}\left(x_{w}, \xi_{i}, t+h\right)=\bar{g}\left(\boldsymbol{x}_{w},-\boldsymbol{\xi}_{i}, t+h\right), \quad \boldsymbol{\xi}_{i} \cdot \boldsymbol{n}>0 .
$$

While for a wall with a fixed temperature $T_{w}$, the boundary condition is [36]

$$
\bar{g}\left(x_{w}, \xi_{i}, t+h\right)=-\bar{g}\left(x_{w},-\boldsymbol{\xi}_{i}, t+h\right)+2 \bar{\omega}_{i} T_{w}, \quad \boldsymbol{\xi}_{i} \cdot \boldsymbol{n}>0 .
$$

\section{Results and discussions}

In this section, several numerical simulations of two-dimensional convective heat transfer problems in porous media at REV scale are conducted to validate the present DUGKS. The test problems include the mixed convection in a porous channel, natural convection in a porous cavity, and the natural convection in a cavity partially filled with porous medium. The algorithm is coded in $C$ programming language and implemented on a cluster (Dual Inter Xeon CPU E5-2680 v3 with 64 GB RAM memory). In the test, certain Cartesian meshes are employed with grid-independent resolutions. In all cases, we set $\sigma=1, \mathrm{Je}=1, g \beta=0.1$. $T_{r e f}$ is set as the average temperature in the flow domain. From Eqs. (3.7) and (3.8), $\tau_{v}$ and $\tau_{c}$ can be determined by $\tau_{v}=\sqrt{\frac{g \beta \Delta T L^{3} P r}{R a}} / R T_{0}$ and $\tau_{c}=\tau_{v} / P r / \sigma$, respectively. Besides, in order to ensure that the flow is nearly incompressible, we set $R T_{0}=10$ to obtain a small Ma $\approx u_{0} / \sqrt{R T_{0}}$, where $u_{0}=\sqrt{g \beta \Delta T H}$ is the characteristic velocity. The time step is given by $\Delta t=\eta \Delta x_{\min } / C$, where $\Delta x_{\min }$ is the minimum grid length, $C$ is the magnitude of the maximal discrete velocity, and the Courant-FriedrichsLewy (CFL) number is set to $\eta=0.5$. For steady-state problems of interest, the following criterion is used,

$$
\sqrt{\frac{\sum_{i, j} \mid\left\|\boldsymbol{u}_{i, j}^{n}-\boldsymbol{u}_{i, j}^{n-1000}\right\|^{2}}{\sum_{i, j}\left\|\boldsymbol{u}_{i, j}^{n}\right\|^{2}}}<10^{-8} \text {. }
$$

\subsection{Mixed convection in a porous channel}

The first test case is the mixed convection in a porous channel of width $H$ and length $L$. The upper plate is held at a fixed temperature $T_{c}$ and moves with a constant velocity $u_{0}$ in the horizontal direction ( $x$-direction), and the static bottom plate is held at a fixed temperature $T_{h}\left(T_{h}>T_{c}\right)$. The fluid is injected through the bottom plate and withdrawn at the same rate $v_{0}$ from the upper one. In this test, an external body force is imposed in vertical direction (y-direction),

$$
a_{y}=\frac{v}{K} v_{0}-g \beta \Delta T\left[\frac{\exp \left(y v_{0} / \alpha_{m}\right)-1}{\exp \left(H v_{0} / \alpha_{m}\right)-1}\right] .
$$


Without the nonlinear force term $\left(F_{\epsilon}=0\right)$, which is considered in our simulations the analytical solutions of this problem can be obtained [21],

$$
\begin{aligned}
& u^{*}=u_{0} \exp \left[r_{1}\left(\frac{y}{H}-1\right)\right] \frac{\sinh \left(r_{2} \cdot y / H\right)}{\sinh \left(r_{2}\right)}, \\
& v^{*}=v_{0} \\
& T^{*}=T_{r e f}+\Delta T \frac{\exp (\operatorname{PrRe} \cdot y / H)-1}{\exp (\operatorname{PrRe})-1},
\end{aligned}
$$

where $\operatorname{Re}$ is the Reynolds number defined by $\operatorname{Re}=H v_{0} / v$, the reference temperature $T_{r e f}=\left(T_{h}+T_{c}\right) / 2, r_{1}$ and $r_{2}$ are given by

$$
r_{1}=\frac{\mathrm{Re}}{2 \epsilon}, \quad r_{2}=\frac{1}{2 \epsilon \mathrm{Je}} \sqrt{\operatorname{Re}^{2}+\frac{4 \epsilon^{3} \mathrm{Je}}{\mathrm{Da}}} .
$$

In our simulations, the periodic boundary conditions are imposed on the inlet and outlet of the porous channel, respectively, and the boundary conditions given by Eq. (3.41) and Eq. (3.43) are imposed on the upper and the bottom walls. The other parameters are set as follows, $H=L=1, \Delta T=T_{h}-T_{c}=1.0, \operatorname{Pr}=1.0, \mathrm{Da}=0.01, \mathrm{Ra}=100, \epsilon=0.4$, and $u_{0}=0.1$. A mesh with uniform spacing in x-direction and non-uniform spacing in $y$ direction is employed in this subsection unless otherwise stated, where the center of cell $\left(x_{i}, y_{j}\right)$ is given by $x_{i}=\frac{L}{N_{x}} \cdot \frac{2 i+1}{2 N_{x}}$, and $i=0,1, \cdots, N_{x}-1, y_{j}=\left(\zeta_{j}+\zeta_{j+1}\right) / 2$, with

$$
\zeta_{j}=0.5+\frac{\tanh \left[a\left(j / N_{y}-0.5\right)\right]}{2 \tanh (a / 2)}, \quad j=0,1, \cdots, N_{y}-1
$$

where $N_{x}$ and $N_{y}$ are the grid number in $x$-direction and $y$-direction, respectively, $a$ is a coefficient which ensures a reasonable mesh distribution.

First, the numerical accuracy of the present method is tested at different Reynolds numbers. A nonuniform $32 \times 32$ mesh is used, with uniform spacing in $x$-direction, and the mesh points in $y$-direction are generated by Eq. (4.5) with $a=2.5$. The minimum cell spacing is thus $3.125 \times 10^{-2}$. The normalized velocity and temperature profiles together with analytical solutions are plotted in Fig. 1. It can be seen that numerical results are in excellent agreement with the analytical solutions.

In what follows, the numerical accuracy of the present method is tested at $\operatorname{Re}=10$. Here, the uniform cells are used for both vertical and horizontal directions, and the grid size $\Delta x$ varies from $1 / 32$ to $1 / 80$. The global relative error $E_{\phi}$ in terminal velocity and temperature fields is defined as

$$
E_{\phi}=\frac{\sqrt{\sum_{i, j}\left|\phi_{i, j}-\phi_{i, j}^{*}\right|^{2}}}{\sqrt{\sum_{i, j}\left|\phi_{i, j}^{*}\right|^{2}}}, \phi=u \text { or } T \text {, }
$$




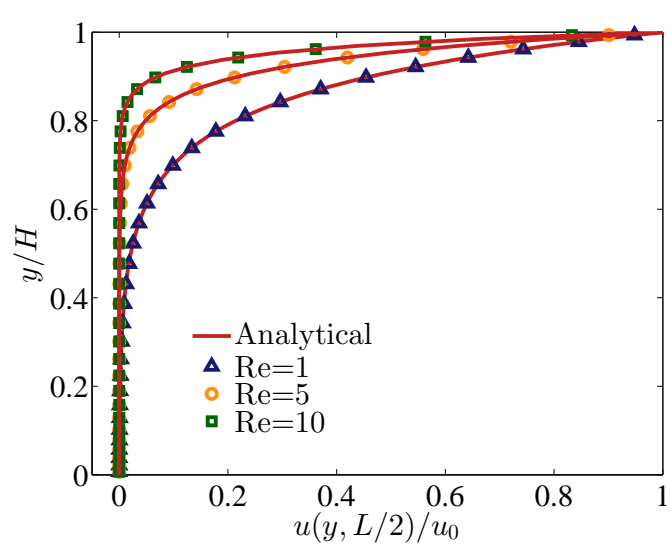

(a)

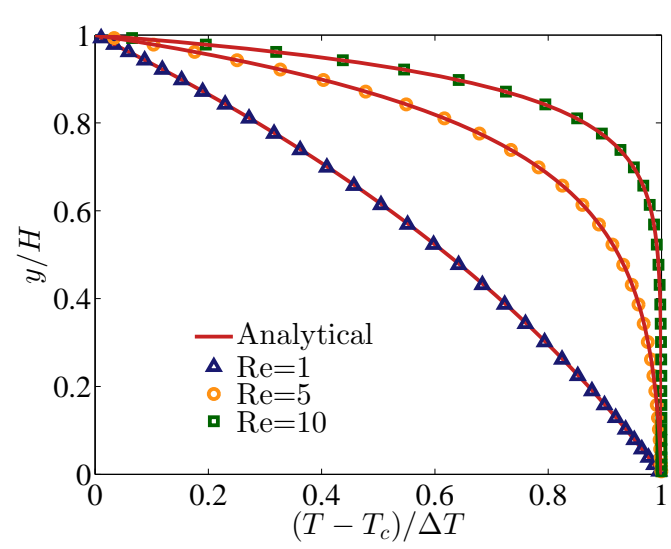

(b)

Fig. 1: Profiles of (a) velocity and (b) temperature of mixed convection for different $\mathrm{Re}$ at $\mathrm{Da}=0.01, \mathrm{Ra}=100$, $\operatorname{Pr}=1.0, \epsilon=0.4$ with a mesh size of $32 \times 32$. Solid lines: analytical solutions; Symbols: numerical results.

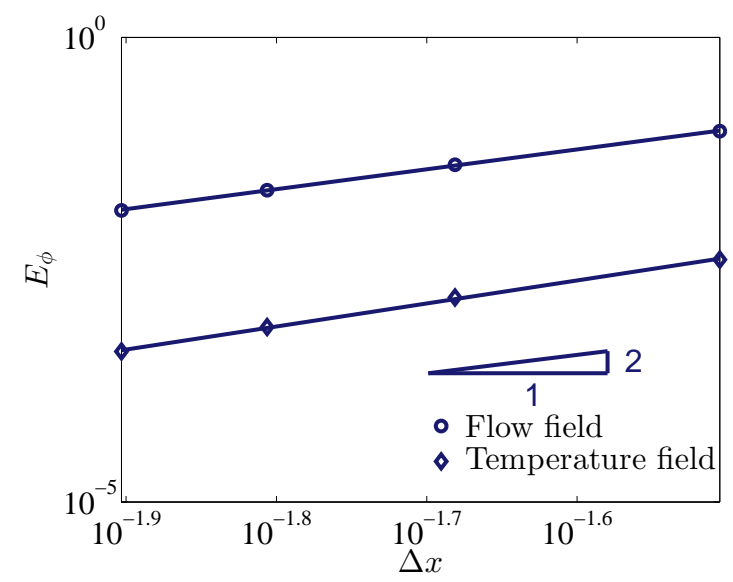

Fig. 2: Global relative errors in velocity and temperature fields against grid size at $\operatorname{Re}=10, \mathrm{Da}=0.01, \mathrm{Ra}=100$, $\operatorname{Pr}=1.0$, and $\epsilon=0.4$. Symbols: numerical results; Solid lines: fitting lines.

Table 1: The global relative errors in velocity and temperature fields.

\begin{tabular}{||ccccc||}
\hline grid size & $32 \times 32$ & $48 \times 48$ & $64 \times 64$ & $80 \times 80$ \\
\hline$E_{u}$ & $9.772868 \times 10^{-2}$ & $4.258826 \times 10^{-2}$ & $2.267580 \times 10^{-2}$ & $1.372878 \times 10^{-2}$ \\
$E_{T}$ & $4.051952 \times 10^{-3}$ & $1.586637 \times 10^{-3}$ & $7.629909 \times 10^{-4}$ & $4.164931 \times 10^{-4}$ \\
\hline
\end{tabular}

where $\phi$ is the numerical solution, $\phi^{*}$ is the analytical solution given by Eq. (4.3). The errors in velocity and temperature fields are shown in Table 1 and plotted in Fig. 2 . It is clear that the present method for flow and temperature fields has second-order spatial accuracy. 

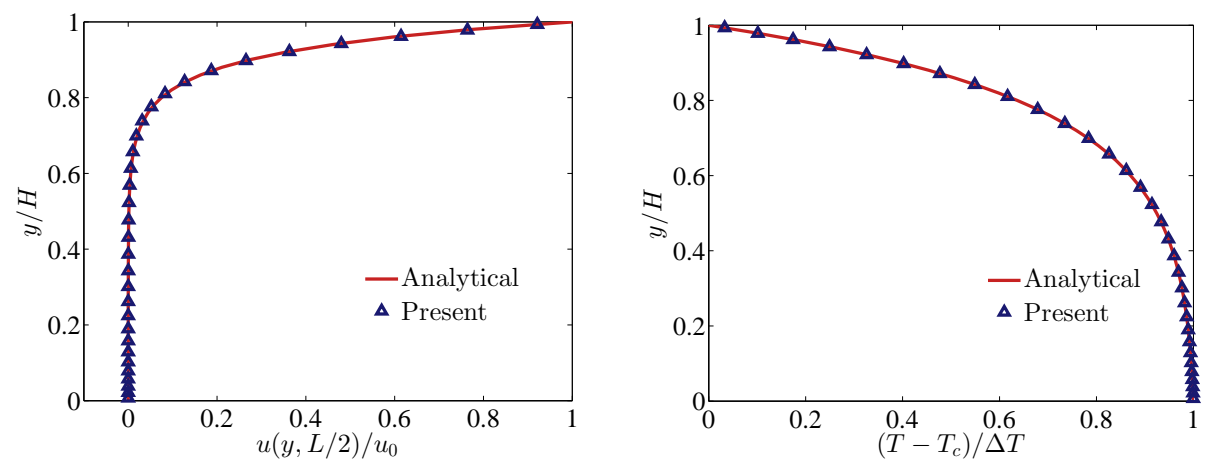

(a)
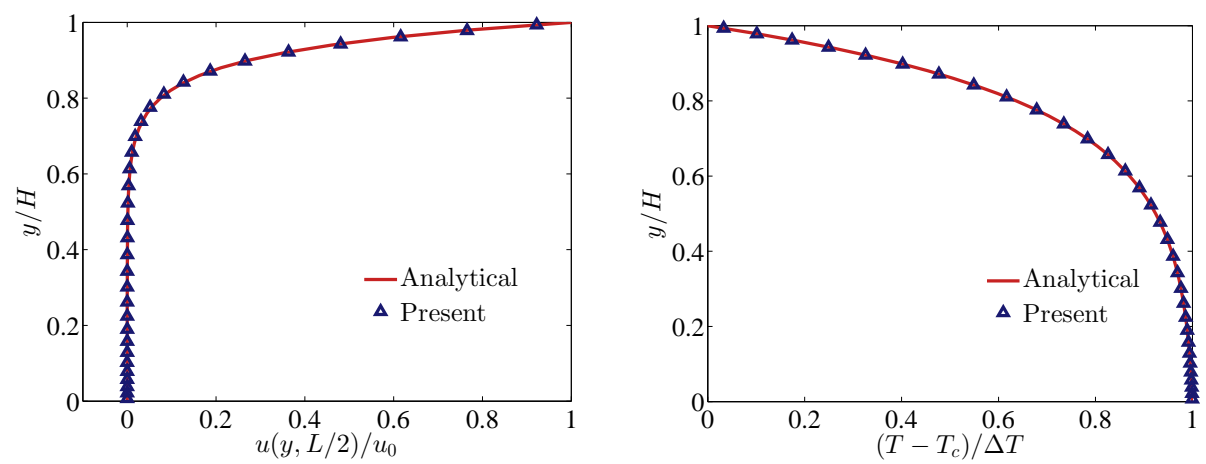

(b)

Fig. 3: Profiles of velocity (left) and temperature (right) of mixed convection with low viscosities (a) $v=5 \times 10^{-4}$ and (b) $v=1.3 \times 10^{-4}$ at $\mathrm{Da}=0.01, \operatorname{Ra}=100, \operatorname{Pr}=1.0, \epsilon=0.6$ with a mesh size of $32 \times 32$.

Furthermore, we performed simulations with low viscosities at $\operatorname{Re}=5, \operatorname{Pr}=1.0, \mathrm{Da}=$ $0.01, \mathrm{Ra}=100$, and $\epsilon=0.6$. A nonuniform $32 \times 32$ mesh is used, with uniform spacing in $x$-direction, and the mesh points in $y$-direction are generated by Eq. (4.5) with $a=2.5$. The minimum cell spacing is thus $3.125 \times 10^{-2}$. In Fig. 3 , the velocity and temperature profiles of the coupled DUGKS with two viscosities $\left(v=5 \times 10^{-4}\right.$ and $\left.v=1.3 \times 10^{-4}\right)$ are plotted. As seen in the figure, the present model can give satisfied results. For comparison, we also simulated this problem with the LBGK model [21] with the same equilibrium distribution function and discrete velocity set, and not shown here. It is found that with the same mesh, numerical instability occurs at $v=5 \times 10^{-4}$ (the same phenomenon can be found in Ref. [22]). The results demonstrate that the DUGKS has better numerical stability than the corresponding LBGK model.

\subsection{Natural convection in a porous cavity}

The classical natural convection in a square porous cavity has been studied extensively as a benchmark solution for numerical methods $[38,49,50]$. The configuration of the prob- 


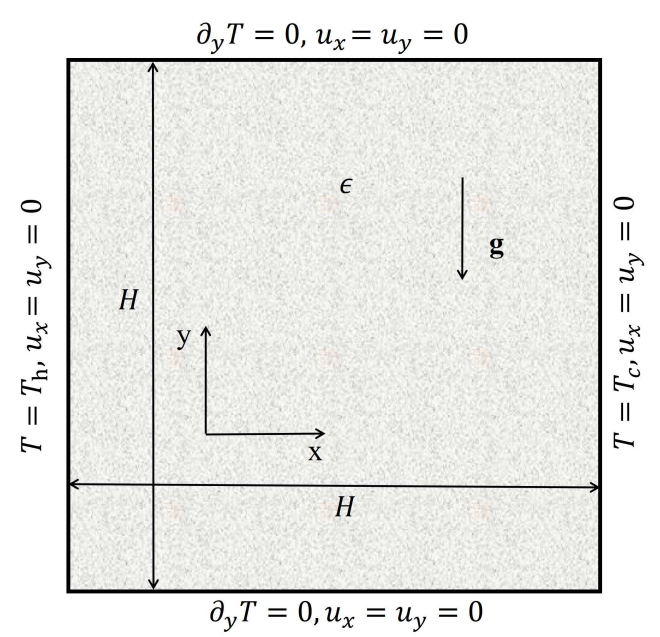

Fig. 4: Geometry of the flow domain and boundary conditions for the natural convection in a porous cavity.

lem is shown Fig. 4, where the upper and lower walls of the cavity are adiabatic, while the left and right walls maintain constant temperatures $T_{h}$ and $T_{c}\left(T_{h}>T_{c}\right)$, respectively, and the height of the cavity is $H=1$. At steady state, the heat transfer can be quantified by the volume average Nusselt number $\overline{\mathrm{Nu}}_{v}$ in the domain or the average Nusselt number $\overline{\mathrm{Nu}}$ of the hot wall,

$$
\begin{aligned}
& \overline{\mathrm{Nu}}_{v}=1+\frac{\langle u T\rangle}{\alpha_{m} \Delta T / H^{\prime}} \\
& \overline{\mathrm{Nu}}=-\int_{0}^{H} \frac{\partial T}{\partial x} d y, \quad x=0,
\end{aligned}
$$

where $u$ is the horizontal velocity and $\langle\cdot\rangle$ represents the average over the whole flow domain.

We first apply the present DUGKS model to the case in which the $\epsilon \rightarrow 1$, and Da tends to infinity with $10^{3} \leq \mathrm{Ra} \leq 10^{6}$. Thus, the convection heat transfer problem actually turns to the case in the absences of porous medium. Both the uniform and the non-uniform meshes are employed for the comparison purpose, and mesh sizes are both set to be $N_{x} \times$ $N_{y}=64 \times 64$. The cell center $\left(x_{i}, y_{j}\right)$ of the non-uniform is generated by $x_{i}=\left(\zeta_{i}+\zeta_{i+1}\right) / 2$ and $y_{j}=\left(\zeta_{j}+\zeta_{j+1}\right) / 2$, where $\zeta_{i}$ and $\zeta_{j}$ are defined by Eq. (4.5) with $i=0,1, \cdots, N_{x}-1$, and $j=0,1, \cdots, N_{y}-1$, respectively. Here, we set $a=4$ in $x$-direction, and $a=2.5$ in $y$-direction, respectively. Numerical results and the benchmark data [46] are shown in Table 2 for $D a=$ $10^{6}, \operatorname{Pr}=0.71$, and $\epsilon=0.9999$. The quantities compared include the maximum horizontal velocity $u_{\max }$ and the corresponding $y$-coordinate $y_{\max }$ on the vertical centerline $(x=$ $H / 2)$, the maximum vertical velocity $v_{\max }$ and the corresponding $x$-coordinate $x_{\max }$ on the horizontal centerline $(y=H / 2)$, and $\overline{\mathrm{Nu}}_{v}$. Here, all the velocities are normalized by the reference velocity $\alpha / H$, and the $x$ - and $y$-coordinates are normalized by $H$. It can be seen that results from both the uniform and non-uniform meshes are in good agreement with 
Table 2: Comparisons of numerical results by present DUGKS with the benchmark solutions [46] for four Ra $\left(D a=10^{6}, \operatorname{Pr}=0.71, \epsilon=0.9999\right.$, and grid size: $\left.64 \times 64\right)$.

\begin{tabular}{|c|c|c|c|c|c|}
\hline $\mathrm{Ra}$ & & $10^{3}$ & $10^{4}$ & $10^{5}$ & $10^{6}$ \\
\hline \multirow{3}{*}{$u_{\max }$} & uniform & 3.6335 & 16.1046 & 34.1831 & 60.8429 \\
\hline & non-uniform & 3.6427 & 16.1224 & 34.6414 & 64.0726 \\
\hline & Ref. [46] & 3.649 & 16.178 & 34.73 & 64.63 \\
\hline \multirow{3}{*}{$y_{\max }$} & uniform & 0.8047 & 0.8203 & 0.8516 & 0.8516 \\
\hline & non-uniform & 0.8187 & 0.8188 & 0.8499 & 0.8499 \\
\hline & Ref. [46] & 0.813 & 0.823 & 0.855 & 0.850 \\
\hline \multirow{3}{*}{$v_{\max }$} & uniform & 3.6799 & 19.4637 & 67.1285 & 205.6321 \\
\hline & non-uniform & 3.6943 & 19.5796 & 68.6006 & 219.7266 \\
\hline & Ref. [46] & 3.697 & 19.617 & 68.59 & 219.36 \\
\hline \multirow{4}{*}{$x_{\max }$} & uniform & 0.1797 & 0.1171 & 0.0703 & 0.0391 \\
\hline & non-uniform & 0.1806 & 0.1121 & 0.0648 & 0.0402 \\
\hline & Ref. [46] & 0.178 & 0.119 & 0.066 & 0.0379 \\
\hline & uniform & 1.1169 & 2.2313 & 4.04373 & 8.4107 \\
\hline \multirow[t]{2}{*}{$\overline{\mathrm{Nu}}_{v}$} & non-uniform & 1.1178 & 2.2432 & 4.5196 & 8.8035 \\
\hline & Ref. [46] & 1.118 & 2.243 & 4.519 & 8.8 \\
\hline
\end{tabular}

the data in Ref. [46], and the results from non-uniform mesh is better. To demonstrate the quantitative difference between results of two meshes, the relative errors in $u_{\max }, v_{\max }$ and $\overline{\mathrm{Nu}}_{v}$ are measured,

$$
\delta=\frac{\left|\Phi-\Phi_{a}\right|}{\left|\Phi_{a}\right|}
$$

where $\Phi$ and $\Phi_{a}$ are the numerical result and benchmark solution in Ref. [46], respectively. The curves of the relative error in $u_{\max }, v_{\max }$ and $\overline{\mathrm{Nu}}_{v}$ are plotted in Fig. 5. It is obvious that the results from the non-uniform mesh are more accurate.

Cases with moderate $\mathrm{Da}$ and $\epsilon$ are also simulated to test the influence of porous media. Fig. 6 shows the streamlines and isotherms predicted by present DUGKS for $\mathrm{Ra}^{*}=\mathrm{Da} \times \operatorname{Ra}=100, \operatorname{Pr}=1$, and $\epsilon=0.4$. Non-uniform meshes with size of $64 \times 64,80 \times 80$ and $96 \times 96$ are adopted for $\mathrm{Da}=10^{-2}, \mathrm{Da}=10^{-4}$, and $\mathrm{Da}=10^{-6}$, respectively. As can be seen from the figure, for a fixed $\mathrm{Ra}^{*}$, the thicknesses of velocity and thermal boundary layers near the vertical walls become thinner with decreasing Da. It is also observed that, as Ra increases, the patters of isotherms change from almost vertical to horizontal in the center of cavity, and converge at the upper right and lower left corners. All these findings agree qualitatively with those reported in the literature [21,38,39].

To quantify the results, the profiles of vertical velocity and temperature along the middle of cavity for moderate $\mathrm{Da}$ at $\mathrm{Ra}^{*}=1000, \operatorname{Pr}=1, \epsilon=0.4$ are plotted in Fig. 7. It can be seen that the vertical velocity always reaches to its peak near the hot and the 


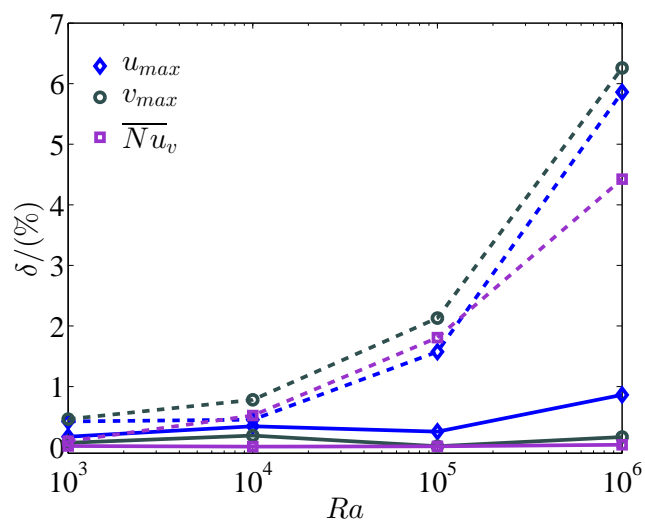

Fig. 5: Relative errors in velocity and the volume average Nusselt number. Solid lines: results of the non-uniform mesh; Dashed lines: results of the uniform mesh.
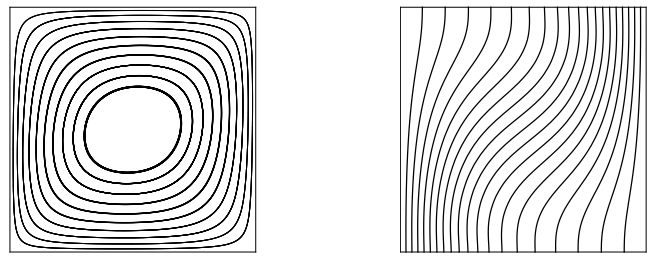

(a)
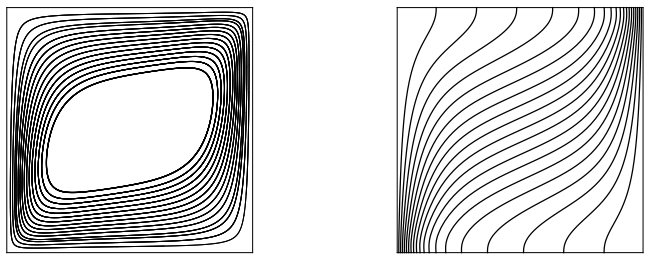

(b)
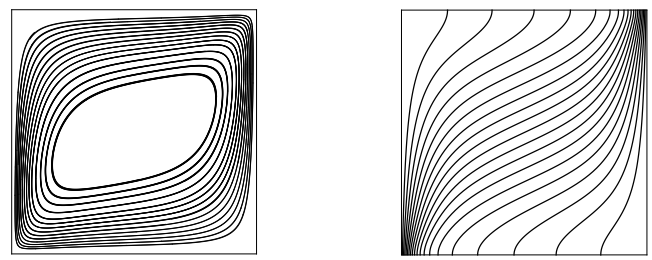

(c)

Fig. 6: Streamlines (left) and isotherms (right) of natural convection in a cavity for $\epsilon=0.4$ and $\operatorname{Pr}=1.0:(a)$ $D a=10^{-2}, R a=10^{4}$; (b) $D a=10^{-4}, R a=10^{6}$; (c) $D a=10^{-6}, R a=10^{8}$. 


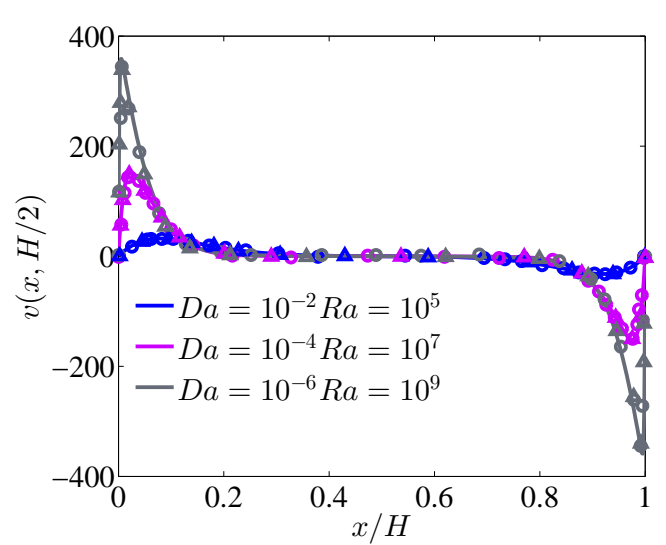

(a)

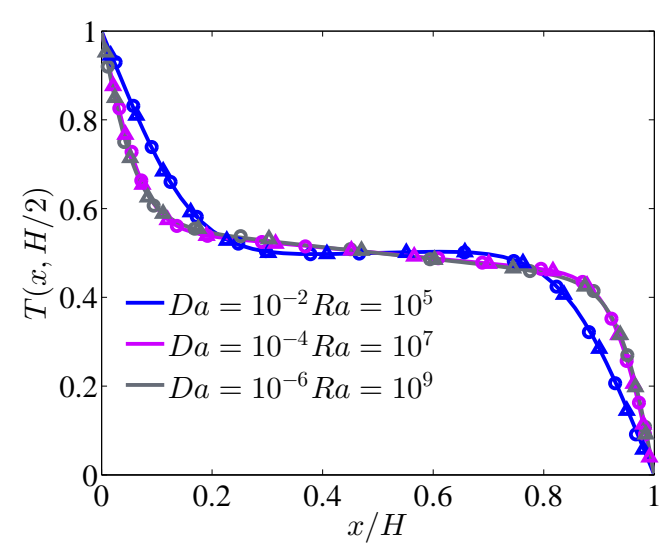

(b)

Fig. 7: Profiles of (a) vertical velocity and (b) temperature at mid-height $(y=H / 2)$. Solid lines: numerical results by present method; Symbols: numerical results by LBM $[21,47]$.

Table 3: Comparisons of the average Nusselt number $\overline{\mathrm{Nu}}$ for moderate $\mathrm{Da}(\operatorname{Pr}=1.0, \epsilon=0.4)$.

\begin{tabular}{||cccccccc||}
\hline \multirow{2}{*}{$D a$} & \multirow{2}{*}{$\mathrm{R} a$} & \multicolumn{3}{c|}{$\epsilon=0.4$} & & \multicolumn{3}{c||}{$\epsilon=0.6$} \\
\cline { 3 - 7 } $10^{-2}$ & $10^{3}$ & 1.007 & 1.008 & 1.008 & 1.012 & 1.012 & 1.012 \\
& $10^{4}$ & 1.362 & 1.365 & 1.360 & 1.494 & 1.498 & 1.490 \\
& $10^{5}$ & 3.009 & 3.012 & 2.990 & 3.460 & 3.463 & 3.435 \\
& $10^{5}$ & 1.067 & 1.067 & 1.064 & 1.069 & 1.069 & 1.067 \\
$10^{-4}$ & $10^{6}$ & 2.630 & 2.618 & 2.600 & 2.733 & 2.734 & 2.700 \\
& $10^{7}$ & 7.808 & 7.811 & 7.751 & 8.457 & 8.506 & 8.543 \\
& $10^{7}$ & 1.085 & 1.089 & 1.077 & 1.089 & 1.094 & 1.077 \\
$10^{-6}$ & $10^{8}$ & 2.949 & 3.014 & 2.980 & 2.957 & 3.035 & 2.992 \\
& $10^{9}$ & 11.610 & 11.733 & 11.591 & 12.092 & 12.149 & 11.995 \\
\hline
\end{tabular}

cold walls. In addition, the lower the Da is, the larger the velocity gradient near the hot and cold walls will be. It is observed that the results of the present method are in good agreement with the results of the lattice Boltzmann method [21,47]. Besides, the average Nusselt numbers $\overline{\mathrm{Nu}}$ for moderate Da are also calculated. As shown in Table 3, it can be found that the present results agree well with the data reported in Refs. [22,39].

\subsection{Natural convection with a porous layer}

In this subsection, the natural convection in a square cavity partially filled with a porous medium of thickness $S$ is studied. Here, we consider the case in which the porous layer lies vertically. The schematic of the problem is illustrated in Fig. 8, with cavity height $H=$ 


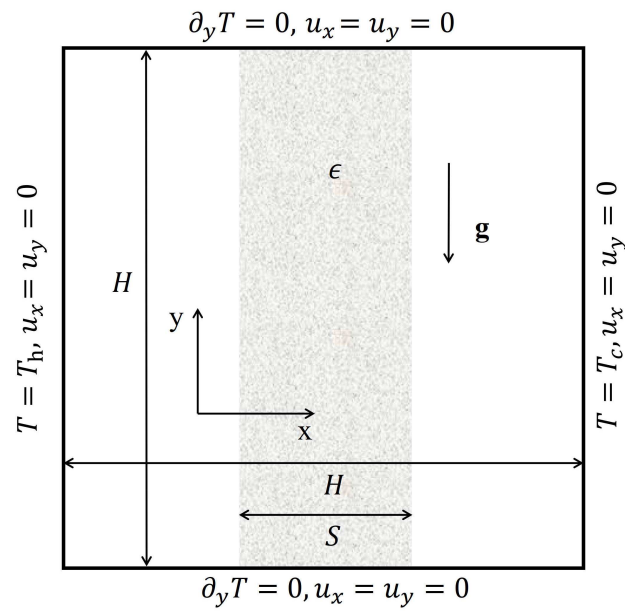

(a)

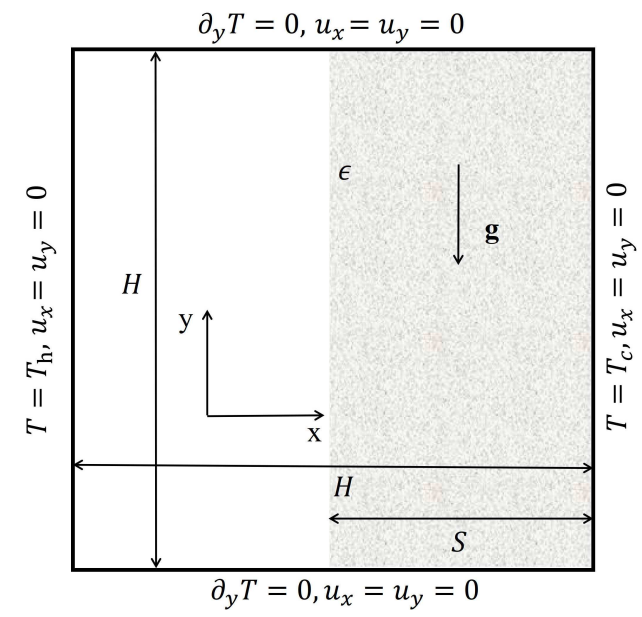

(b)

Fig. 8: Geometry of the flow domain and boundary conditions of the natural convection in a square partially filled with porous medium: (a) $S=1 / 3 H$; (b) $S=1 / 2 H$.

Table 4: Test conditions of cases for the porous layer problem.

\begin{tabular}{||cccccccc||}
\hline Case & $S / H$ & Beads $\left(d_{p} \mathrm{~mm}\right)$ & $R a$ & $D a$ & $P r$ & $\alpha_{m}$ & $F_{\epsilon}$ \\
\hline 1 & $1 / 3$ & 6.0 glass & $3.7 \times 10^{6}$ & $1.37 \times 10^{-5}$ & 6.44 & 1.362 & 0.554 \\
2 & $1 / 3$ & $6.35 \mathrm{Al}$ & $3.7 \times 10^{6}$ & $1.534 \times 10^{-5}$ & 6.44 & 37.47 & 0.554 \\
3 & $1 / 2$ & 1.6 glass & $3.028 \times 10^{7}$ & $7.354 \times 10^{-7}$ & 6.97 & 1.397 & 0.6124 \\
4 & $1 / 2$ & 6.0 glass & $3.028 \times 10^{7}$ & $1.296 \times 10^{-5}$ & 6.97 & 1.383 & 0.5647 \\
\hline
\end{tabular}

1. The boundary conditions are the same as the natural convection problem in Section 4.2. The porous medium is consisted of random packing of spherical glass or aluminum (Al) beads. We use water as the test fluid, and the other governing parameters for four test cases are listed in Table 4. In Case 1 and Case 2, the porous layer is put in the middle of the cavity; In Case 3 and Case 4, the porous layer is put in the right of the cavity. The mesh size is $N_{x} \times N_{y}=120 \times 120$, with progressively finer grids near the vertical walls and porous-fluid interface. In Case 1 and Case 2, the cell center $\left(x_{i}, y_{j}\right)$ is generated by $x_{i}=\left(\zeta_{i}+\zeta_{i+1}\right) / 2$ for $i=0$ to $39, x_{i}=x_{i-40}+H / 3$ for $i=40$ to 79 , and $x_{i}=x_{i-40}+H / 3$ for $i=80$ to $119, y_{j}=\frac{H}{N_{y}} \cdot \frac{2 j+1}{2 N_{y}}$, for $j=0,1, \cdots, N_{y}-1$, where $\zeta_{i}$ is defined by Eq. (4.5) with $a=3$. Therefore, the minimum grid spacing is $2.67 \times 10^{-3}$. In Case 3 and Case 4 , the cell center $\left(x_{i}, y_{j}\right)$ is generated by $x_{i}=\left(\zeta_{i}+\zeta_{i+1}\right) / 2$ for $i=0$ to 59 , and $x_{i}=x_{i-60}+H / 2$ for $i=60$ to 119 , while $y_{j}=\frac{H}{N_{y}} \times \frac{2 j+1}{2 N_{y}}$ for $j=0,1, \cdots, N_{y}-1$, where $\zeta_{i}$ is defined by Eq. (4.5) with $a=3$, and thus the minimum grid spacing is $2.61 \times 10^{-3}$.

Streamlines and isotherms predicted by DUGKS in Case 1 and Case 2 are shown in Fig. 9. As seen in the figure, there are two eddies in the left and right fluid layers in 

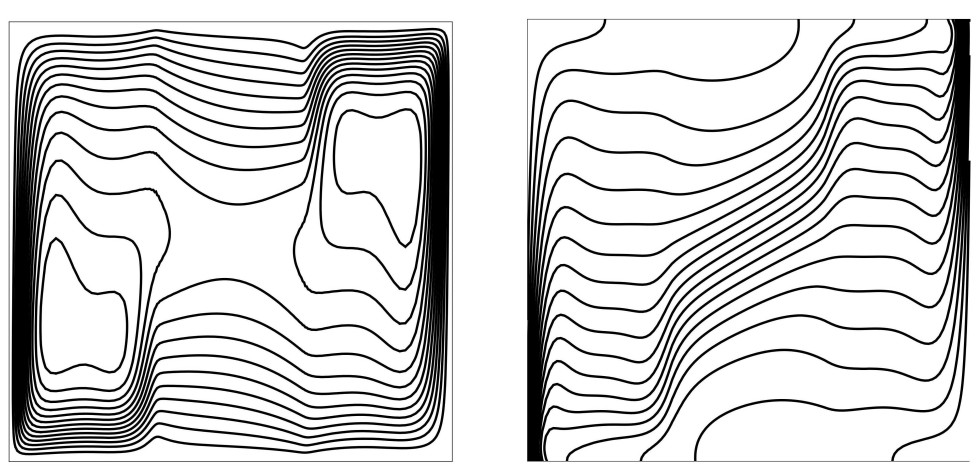

(a)
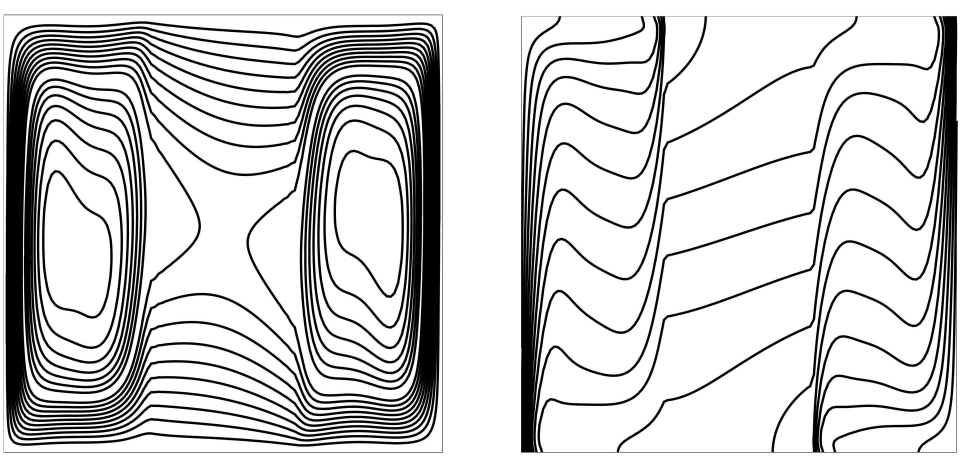

(b)

Fig. 9: Predicted streamlines (left) and isotherms (right) of natural convection in a square with a porous layer: (a) Case 1; (b) Case 2.

Table 5: The maximum value of the stream function $\psi_{\max }$.

\begin{tabular}{||ccccc||}
\hline Case & Ref. [21] & Ref. [49] & Ref. [51] & Present \\
\hline 1 & 18.68 & 16.4 & 16.52 & 16.77 \\
2 & 24.93 & 23.9 & 23.21 & 23.47 \\
\hline
\end{tabular}

two cases, and in Case 1, the two eddies move toward the upper right and lower left corners. As for the case of the aluminum beads (Case 2), the isotherms show large variations in slope at the fluid-porous interface, which can be attributed to a high effective thermal conductivity of aluminum. All of these observations are qualitatively consistent with those of previous experimental and numerical results $[49,51]$. Furthermore, the maximum values of stream function $\psi_{\max }$ of Case 1 and Case 2 are listed in Table 5, which show a good agreement between the present predictions and the results in Refs. [21,49,51].

We now examine the Case 3 and Case 4. The flow and thermal patterns are similar to those reported in [48], and not shown here. The temperature distributions at three 


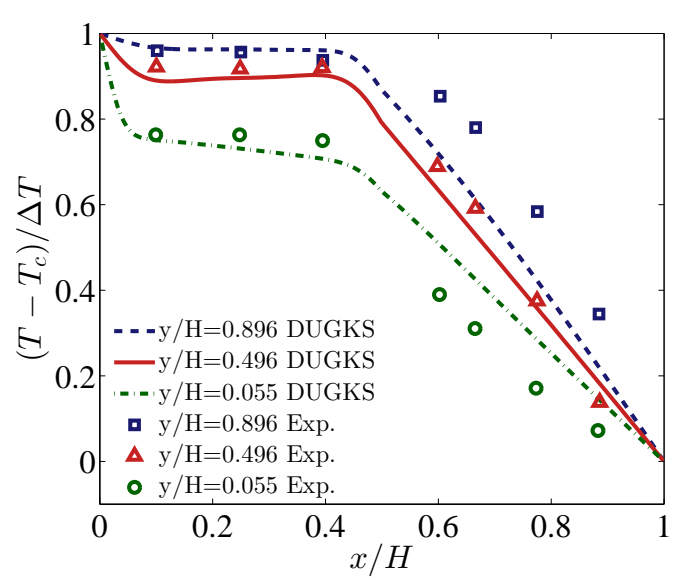

(a)

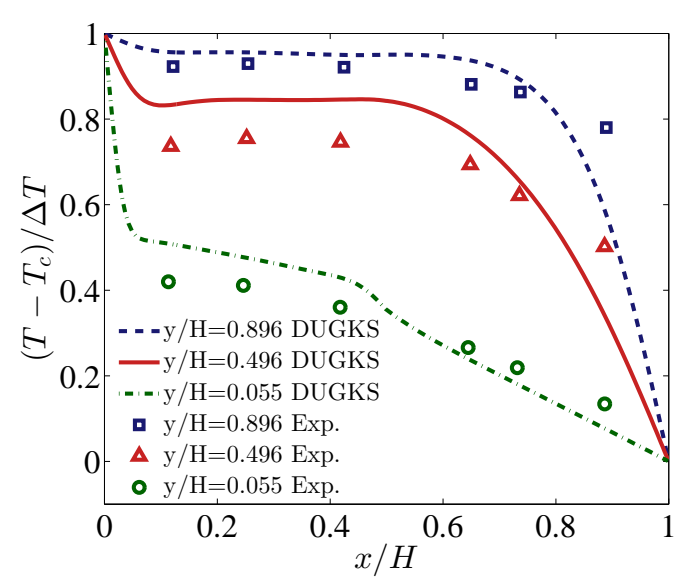

(b)

Fig. 10: The temperature distributions at three locations: (a) Case 3; (b) Case 4. Solid lines: results predicted by present DUGKS; Symbols: experimental data [48].

locations predicted by the DUGKS and the experimental results [48] are plotted in Fig. 10. Again, the present numerical results are in good agreement with the experimental data.

\section{Conclusion}

In this work, a coupled discrete unified gas kinetic scheme is developed for simulating the incompressible fluid flow and heat transfer convection in porous media at REV scale. In this method, the velocity field and the temperature field are described by two DUGKS models, and are coupled with the Boussinesq assumption. The effects of porous media are incorporated by introducing the porosity into equilibrium distribution function and adding the flow resistance force in the kinetic equation for velocity field.

The method is well validated by simulating three two-dimensional convection problems in porous media. The numerical results are in good agreement with previous numerical and/or experimental results. It is also shown that the present DUGKS is of second order accuracy in space. Furthermore, compared with the corresponding LBGK model, the present model has better numerical stability. Furthermore, non-uniform meshes can be employed to improve the computational efficiency. In summary, the present coupled DUGKS can serve as an ideal numerical tool to study the convection heat transfer in porous media at the REV scale.

\section{Acknowledgments}

The authors would like to thank the support by the National Natural Science Foundation of China (No. 11872024). 


\section{Nomenclature}

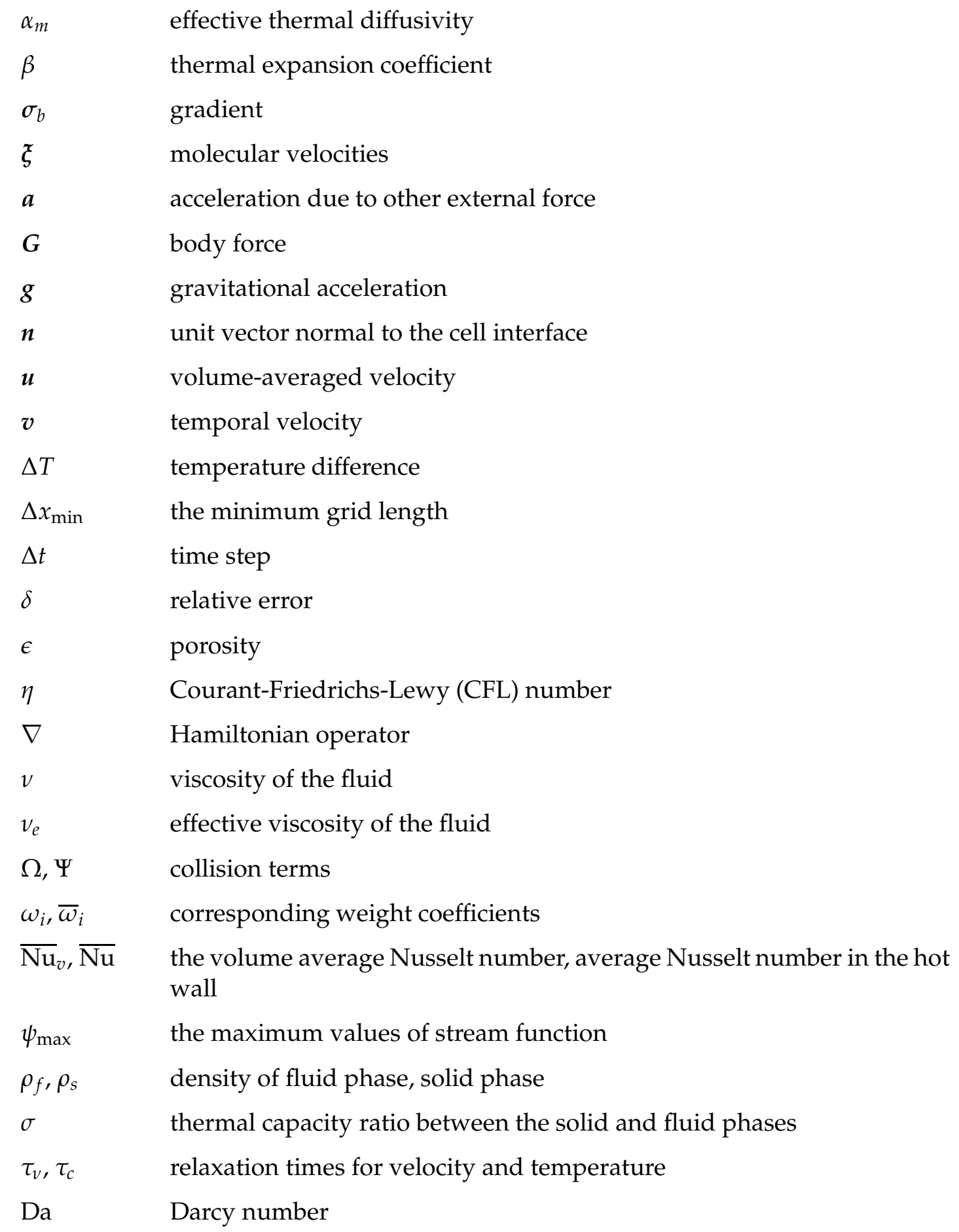




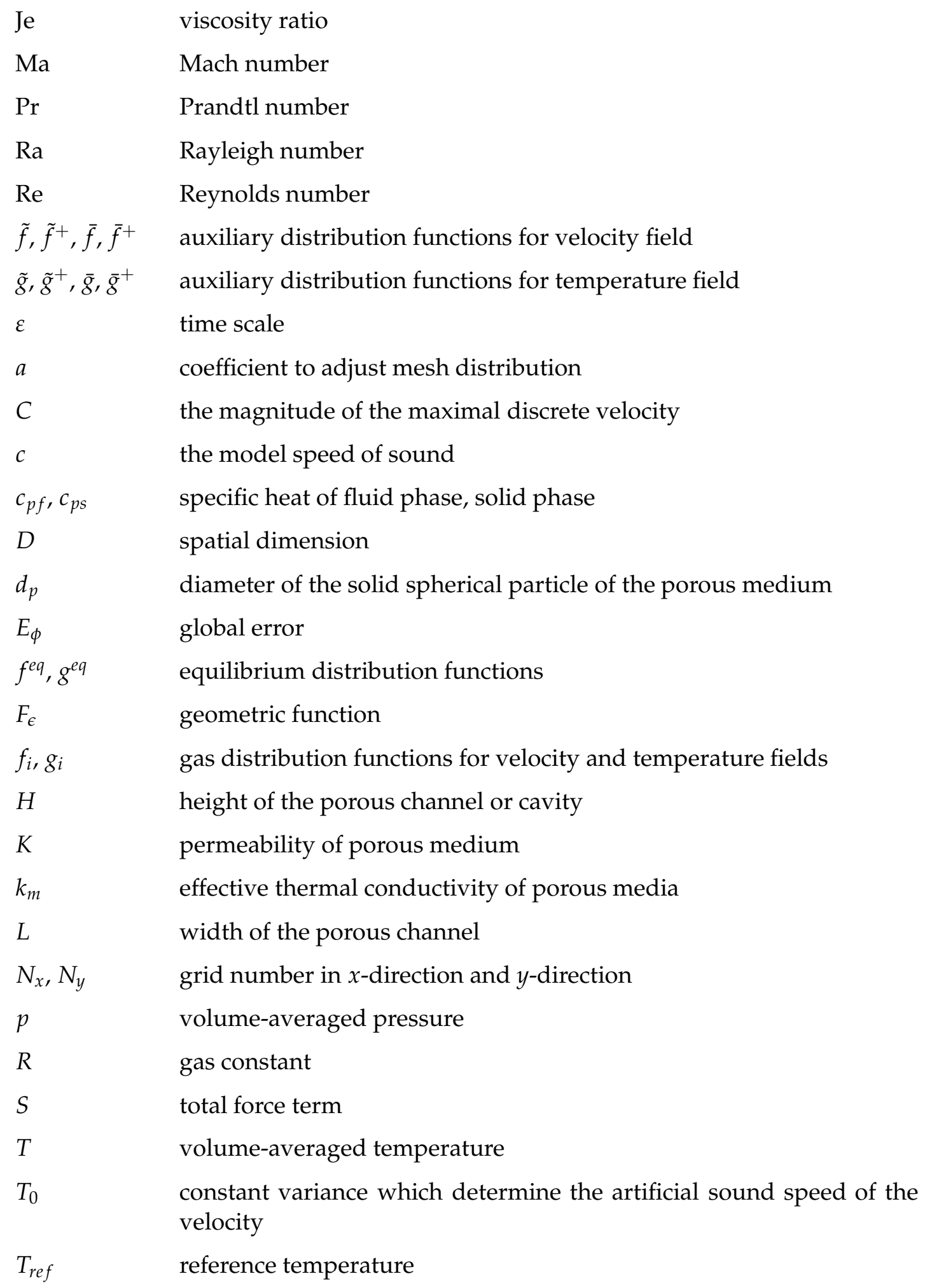


$u_{0} \quad$ characteristic velocity

$u_{\max }, y_{\max } \quad$ the maximum horizontal velocity and the corresponding $y$-coordinate

$v_{\max }, x_{\max } \quad$ the maximum vertical velocity and the corresponding $x$-coordinate

\section{Appendix: Chapman-Enskog analysis}

The Chapman-Enskog analysis is adopted to recover the macroscopic equation (2.1) from Eqs. (3.1) and (3.2). To this end, the following multiscale expansions in space and time are adopted,

$$
\begin{aligned}
& f=f^{(0)}+\varepsilon f^{(1)}+\varepsilon^{2} f^{(2)}, \\
& \partial_{t}=\varepsilon \partial_{t_{0}}+\varepsilon^{2} \partial_{t_{1}}, \quad \nabla=\varepsilon \nabla_{0}, \quad S=\varepsilon S^{(0)} .
\end{aligned}
$$

By substituting them into Eq. (3.1), we can obtain

$$
\begin{array}{ll}
\mathcal{O}\left(\varepsilon^{0}\right): & f^{(0)}=f^{e q}, \\
\mathcal{O}\left(\varepsilon^{1}\right): & \partial_{t_{0}} f^{(0)}+\mathcal{\zeta} \cdot \nabla_{0} f^{0}=-\frac{1}{\tau_{v}} f^{(1)}+S^{(0)} \\
\mathcal{O}\left(\varepsilon^{2}\right): & \partial_{t_{0}} f^{(1)}+\xi \cdot \nabla_{0} f^{(1)}+\partial_{t_{1}} f^{(0)}=-\frac{1}{\tau_{v}} f^{(2)} .
\end{array}
$$

From the equilibrium distribution function defined by Eq. (3.23), we can obtain

$$
\begin{aligned}
& \int f^{e q} d \xi=\rho, \quad \int \xi f^{e q} d \xi=\rho \boldsymbol{u}, \quad \int \xi \xi f^{e q} d \xi=\rho \boldsymbol{u} \boldsymbol{u} / \epsilon+p \epsilon, \\
& \int \xi \xi \xi f^{e q}=\rho R T_{0}\left(u_{\alpha} \delta_{\beta \gamma}+u_{\beta} \delta_{\alpha \gamma}+u_{\gamma} \delta_{\alpha \beta}\right), \\
& \int S^{(0)} d \xi=0, \quad \int \xi S^{(0)} d \boldsymbol{\xi}=\boldsymbol{F}, \quad \int \xi \xi S^{(0)} d \boldsymbol{\xi}=\boldsymbol{F} \boldsymbol{u}+\boldsymbol{u} \boldsymbol{F},
\end{aligned}
$$

where $p=\rho R T_{0} / \epsilon$ is the pressure. Then, taking the zeroth- to second-order moments of Eq. (A.4), respectively, we can get the following equations on the $t_{0}$ scale,

$$
\begin{aligned}
& \partial_{t_{0}} \rho+\nabla_{x_{0}} \cdot(\rho \boldsymbol{u})=0, \\
& \partial_{t_{0}}(\rho \boldsymbol{u})+\nabla_{x_{0}} \cdot(\rho \boldsymbol{u} \boldsymbol{u} / \epsilon+p \epsilon)=\boldsymbol{F}, \\
& \partial_{t_{0}}(\rho \boldsymbol{u} \boldsymbol{u} / \epsilon+p \epsilon)+\nabla_{x_{0}} \cdot\left(\rho R T_{0}\left(u_{\alpha} \delta_{\beta \gamma}+u_{\beta} \delta_{\alpha \gamma}+u_{\gamma} \delta_{\alpha \beta}\right)\right)=-\frac{1}{\tau_{v}} \int \xi \xi f^{(1)} d \boldsymbol{\xi}+\boldsymbol{F} \boldsymbol{u}+\boldsymbol{u} \boldsymbol{F} .
\end{aligned}
$$

Similarly, taking the zeroth- and first-order moments of Eq. (A.5),

$$
\begin{aligned}
& \partial_{t_{1}} \rho=0, \\
& \partial_{t_{1}} \rho \boldsymbol{u}+\nabla_{0} \cdot \int \xi \xi f^{(1)} d \xi=0 .
\end{aligned}
$$


Combing Eqs. (A.9) and (A.12), we can get

$$
\partial_{t} \rho+\nabla(\rho \boldsymbol{u})=0
$$

Note that for incompressible flows, Eq. (A.14) can be written as

$$
\nabla \cdot \boldsymbol{u}=0
$$

According to Eq. (A.11), the second moment of $f^{(1)}$ can be rewritten as

$$
\int \xi \xi f^{(1)} d \boldsymbol{\xi}=-\tau_{c} R T_{0} \rho\left(\nabla \boldsymbol{u}+\nabla \boldsymbol{u}^{T}\right)+\mathcal{O}\left(M a^{3}\right) .
$$

For the above equation, the terms of order $\mathcal{O}\left(u^{3}\right)$ or higher have been neglected. Then, substituting Eq. (A.16) into Eq. (A.13), we can get

$$
\partial_{t_{1}} \rho \boldsymbol{u}-\tau_{c} R T_{0} \rho \nabla_{0} \cdot\left(\nabla \boldsymbol{u}+\nabla \boldsymbol{u}^{T}\right)=0 .
$$

For incompressible flows, Eqs. (A.10) and (A.17) are lead to

$$
\partial_{t}(\boldsymbol{u})+\nabla \cdot\left(\frac{\boldsymbol{u} \boldsymbol{u}}{\epsilon}\right)=-\frac{1}{\rho} \nabla(\epsilon p)+v_{e} \nabla \cdot\left(\nabla \boldsymbol{u}+\nabla \boldsymbol{u}^{T}\right)+\boldsymbol{F},
$$

where $v_{e}=\tau_{c} R T_{0}$ is the effective viscosity, $\rho$ is the density of fluid, and $\nabla \cdot(\nabla \boldsymbol{u})^{T}=\nabla(\nabla \cdot$ $\boldsymbol{u})=0$. From these treatments, Eq. (A.18) can recover Eq. (2.1a),

$$
\partial_{t}(\boldsymbol{u})+\nabla \cdot\left(\frac{\boldsymbol{u} \boldsymbol{u}}{\epsilon}\right)=-\frac{1}{\rho} \nabla(\epsilon p)+v_{e} \nabla^{2} \boldsymbol{u}+\boldsymbol{F} .
$$

Next, the temperature equation will be derived based on Eq. (3.2). Similarly, the distribution function is expanded as

$$
g=g^{(0)}+\varepsilon g^{(1)}+\varepsilon^{2} g^{(2)} .
$$

By substituting it into Eq. (3.2) and equalling with respect to the same order of $\varepsilon$, we can obtain

$$
\begin{array}{ll}
\mathcal{O}\left(\varepsilon^{0}\right): & g^{(0)}=g^{e q} \\
\mathcal{O}\left(\varepsilon^{1}\right): & \partial_{t_{0}} g^{(0)}+\mathcal{\zeta} \cdot \nabla_{0} g^{0}=-\frac{1}{\tau_{c}} g^{(1)} \\
\mathcal{O}\left(\varepsilon^{2}\right): & \partial_{t_{0}} g^{(1)}+\mathcal{\xi} \cdot \nabla_{0} g^{(1)}+\partial_{t_{1}} g^{(0)}=-\frac{1}{\tau_{c}} g^{(2)} .
\end{array}
$$

According to Eq. (3.23), we can obtain

$$
\int g^{e q} d \xi=\sigma T, \quad \int \xi g^{e q} d \xi=u T, \quad \int \xi \xi g^{e q} d \xi=R T_{0} \sigma T .
$$


Then, taking the zeroth- and first-order moments of Eqs. (A.22) and (A.23), we can obtain

$$
\begin{aligned}
& \partial_{t_{0}}(\sigma T)+\nabla_{0} \cdot(\boldsymbol{u} T)=0, \\
& \partial_{t_{0}}(\boldsymbol{u} T)+R T_{0} \nabla_{0} \cdot(\sigma T)=-\frac{1}{\tau_{c}} \int \boldsymbol{\xi}^{(1)} d \boldsymbol{\xi}, \\
& \partial_{t_{1}}(\sigma T)+\int \xi \cdot \nabla_{0} g^{(1)}=0 .
\end{aligned}
$$

After substituting Eqs. (A.26) and (A.27), we get

$$
\partial_{t_{1}}(\sigma T)-\tau_{c} \nabla_{0} \cdot\left[\partial_{t_{0}}(\boldsymbol{u} T)+R T_{0} \nabla_{0} \cdot(\sigma T)\right]=0 .
$$

Combing the macroscopic equations at two time scales without the higher terms, we finally obtain the following equation

$$
\frac{\partial(\sigma T)}{\partial t}+\nabla \cdot(\boldsymbol{u} T)=\tau_{c} R T_{0} \nabla \cdot(\nabla(\sigma T)) .
$$

In this work we assume that $\sigma$ does not change with space and time, and then we can get the macroscopic equation

$$
\sigma \frac{\partial(T)}{\partial t}+\nabla \cdot(\boldsymbol{u} T)=\alpha_{m} \nabla \cdot(\nabla T)
$$

where $\alpha_{m}=\sigma \tau_{c} R T_{0}$ is the effective thermal diffusivity.

\section{References}

[1] Y. Varol and H. F. Oztop, A comparative numerical study on natural convection in inclined wavy and at-plate solar collectors, Build. Environ., 43 (2008), 1535-1544.

[2] S. Hirasawa, R. Tsubota, T. Kawanami, and K. Shirai, Reduction of heat loss from solar thermal collector by diminishing natural convection with high-porosity porous medium, Sol. Energy, 97 (2013), 305-313.

[3] A. Revil, H. Schwaeger, L. Cathles III, and P. Manhardt, Streaming potential in porous media: 2. theory and application to geothermal systems, J. Geophys. Res.-Solid Earth, 104 (1999), 20033-20048.

[4] Y. Ho, J. Ng, and G. McKay, Kinetics of pollutant sorption by biosorbents, Sep. Purif. Methods, 29 (2000), 189-232.

[5] H. P. G. Darcy, Les Fontaines publiques de la ville de Dijon. Exposition et application des principes à suivre et des formules à employer dans les questions de distribution d'eau, etc. V. Dalamont, 1856.

[6] D. Gartling, C. Hickox, and R. Givler, Simulation of coupled viscous and porous flow problems, Int. J. Comput. Fluid Dyn., 7 (1996), 23-48.

[7] F. Arpino, G. Cortellessa, and A. Mauro, Transient thermal analysis of natural convection in porous and partially porous cavities, Int. J. Heat Mass Transf., 67 (2015), 605-631.

[8] H. Brinkman, A calculation of the viscous force exerted by a flowing fluid on a dense swarm of particles, Flow, Turbul. Combust., 1 (1949), 27. 
[9] A. S. Joodi, S. Sizaret, S. Binet, A. Bruand, P. Alberic, and M. Lepiller, Development of a Darcy-Brinkman model to simulate water ow and tracer transport in a heterogeneous karstic aquifer," Hy-drogeol. J., 18 (2010), 295-309.

[10] S. Whitaker, The Forchheimer equation: a theoretical development, Transp. Porous Media, 25 (1996), 27-61.

[11] P. Venkataraman and P. R. M. Rao, Validation of Forchheimer's law for flow through porous mediawith converging boundaries, J. Hydraul. Eng., 126 (2000), 63-71.

[12] K. Vafai, Convective ow and heat transfer in variable-porosity media, J. Fluid Mech, 147 (1984), 233-259.

[13] C. Hsu and P. Cheng, Thermal dispersion in a porous medium, Int. J. Heat Mass Transf., 33 (1990), 1587-1597.

[14] D. B. Ingham and I. Pop, Transport phenomena in porous media, Elsevier, 1998.

[15] D. A. Nield, A. Bejan, et al., Convection in porous media, Springer, New York, 2006.

[16] K. Vafai, Handbook of porous media, Crc Press, 2015.

[17] F. Arpino, A. Carotenuto, M. Ciccolella, G. Cortellessa, N. Massarotti, and A. Mauro, Transient natural convection in partially porous vertical annuli, Int. J. Heat Technol., 34 (2016), S512-S518.

[18] Q. Kang, D. Zhang, and S. Chen, Unified lattice Boltzmann method for flow in multiscale porous media, Phy. Rev. E, 66 (2002), 056307.

[19] Z. Guo and T. S. Zhao, Lattice Boltzmann model for incompressible flows through porous media, Phys. Rev. E, 66 (2002), 036304.

[20] Q. Liu and Y. He, Double multiple-relaxation-time lattice Boltzmann model for solid-liquid phase change with natural convection in porous media, Physica A, 438 (2015), 94-106.

[21] Z. Guo and T. Zhao, A lattice Boltzmann model for convection heat transfer in porous media, Numer Heat Tranf. B-Fundam., 47 (2005), 157-177.

[22] Q. Liu, Y. He, Q. Li, and W. Tao, A multiple-relaxation-time lattice Boltzmann model for convection heat transfer in porous media, Int. J. Heat Mass Transf., 73 (2014), 761-775.

[23] Q. Liu, X. Feng, Y. He, C. Lu, and Q. Gu, Three-dimensional multiple-relaxation-time lattice Boltzmann models for single-phase and solid-liquid phase-change heat transfer in porous media at the REV scale, Appl. Therm. Eng., 152 (2019), 319-337.

[24] A. Zarghami, S. D. Francesco, and C. Biscarini, Porous substrate effects on thermal flows through a REV-scale finite volume lattice Boltzmann model, Int. J. Mod. Phys. C, 25 (2014), 1350086.

[25] P. Wang, L. Zhu, Z. Guo, and K. Xu, A comparative study of LBE and DUGKS methods for nearly incompressible flows, Commun. Comput. Phys., 17 (2015), 657-681.

[26] Z. Guo, K. Xu, and R. Wang, Discrete unified gas kinetic scheme for all knudsen number flows: Low-speed isothermal case, Phys. Rev. E, 88 (2013), 033305.

[27] Z. Guo, R. Wang, and K. Xu, Discrete unified gas kinetic scheme for all knudsen number flows. II. Thermal compressible case, Phys. Rev. E, 91 (2015), 033313.

[28] Z. Guo and K. Xu, Discrete unified gas kinetic scheme for multiscale heat transfer based on the phonon Boltzmann transport equation, Int. J. Heat Mass Transf., 102 (2016), 944-958.

[29] D. Pan, C. Zhong and C. Zhuo, An Implicit Discrete Unified Gas-Kinetic Scheme for Simulations of Steady Flow in All Flow Regimes, Commun. Comput. Phys., 25 (2019), pp. 14691495.

[30] Y. Wang, C. Zhong, and S. Liu, Arbitrary Lagrangian-Eulerian-type discrete unified gas kinetic scheme for low-speed continuum and rarefied flow simulations with moving boundaries. Phy. Rev. E, 100 (2019), 063310. 
[31] L. Zhu, Z. Guo, and K. Xu, Discrete unified gas kinetic scheme on unstructured meshes, Comput. Fluids, 127 (2016), 211-225.

[32] L. Zhu and Z. Guo, Application of discrete unified gas kinetic scheme to thermally induced nonequilibrium flows, Comput. Fluids, 193 (2019), 103613.

[33] L. Zhu, P. Wang, and Z. Guo, Performance evaluation of the general characteristics based off-lattice Boltzmann scheme and DUGKS for low speed continuum flows, J. Comput. Phys., 333 (2017), 227-246.

[34] P. Wang, L. Wang, and Z. Guo, Comparison of the lattice Boltzmann equation and discrete unified gas-kinetic scheme methods for direct numerical simulation of decaying turbulent flows, Phy. Rev. E, 94 (2016), 043304.

[35] Y. Bo, P. Wang, Z. Guo, and L. Wang, DUGKS simulations of three-dimensional Taylor-Green vortex flow and turbulent channel flow, Comput. Fluids, 155 (2017), 9-21.

[36] P. Wang, S. Tao, and Z. Guo, A coupled discrete unified gas-kinetic scheme for Boussinesq flows, Comput. Fluids, 120 (2015), 70-81.

[37] P. Wang, Y. Zhang, and Z. Guo, Numerical study of three-dimensional natural convection in a cubical cavity at high Rayleigh numbers, Int. J. Heat Mass Transf., 113 (2017), 217-228.

[38] P. Nithiarasu, K. Seetharamu, and T. Sundararajan, Natural convective heat transfer in a fluid saturated variable porosity medium, Int. J. Heat Mass Transfer, 40 (1997), 3955-3967.

[39] L. Wang, J. Mi, and Z. Guo, A modified lattice Bhatnagar-Gross-Krook model for convection heat transfer in porous media, Int. J. Heat Mass Transf., 94 (2016), 269-291.

[40] G. Neale and W. Nader, Practical significance of Brinkman's extension of Darcy's law: Coupled parallel flows within a channel and a bounding porous medium, Can. J. Chem. Engng, 52 (1974), 475-478.

[41] S. Ergun, Fluid flow through packed columns, Chem. Eng. Prog., 48 (1952), 89-94.

[42] X. Shan, Simulation of Rayleigh-bénard convection using a lattice Boltzmann method, Phys. Rev. E, 55 (1997), 2780.

[43] Y. Qian, D. d'Humières, and P. Lallemand, Lattice BGK models for Navier-Stokes equation, EPL (Europhysics Letters), 17 (1992), 479.

[44] L. Li, R. Mei, J. F. Klausner, Lattice Boltzmann models for the convection-diffusion equation: D2Q5 vs D2Q9, Int. J. Heat Mass Transf., 108(2017), 41-62.

[45] H. Huang, X. Lu, and M. Sukop, Numerical study of lattice boltzmann methods for a convection-diffusion equation coupled with Navier-Stokes equations, Physics A, 44(2011), 055001.

[46] G. de Vahl Davis, Natural convection of air in a square cavity: a bench mark numerical solution, Int. J. Numer. Methods Fluids, 3 (1983), 249-264.

[47] Q. Liu and Y. He, Lattice Boltzmann simulations of convection heat transfer in porous media, Physica A, 465 (2017), 742-753.

[48] C. Beckermann, S. Ramadhyani, and R. Viskanta, Natural convection flow and heat transfer between a fluid layer and a porous layer inside a rectangular enclosure, J. Heat Transf., 109 (1987), 363-370.

[49] C. Beckermann, R. Viskanta, and S. Ramadhyani, Natural convection in vertical enclosures containing simultaneously fluid and porous layers, J. Fluid Mech, 186 (1988), 257-284.

[50] T. Seta, E. Takegoshi, and K. Okui, Lattice Boltzmann simulation of natural convection in porous media, Math. Comput. Simul., 72 (2006), 195-200.

[51] D. Gao, Z. Chen, L. Chen, and D. Zhang, A modified lattice Boltzmann model for conjugate heat transfer in porous media, Int. J. Heat Mass Transf., 105 (2017), 673-683. 